\title{
Resource implications of adopting a restrictive neonatal blood transfusion policy
}

M C Harrison, MB ChB, MRCP, FRCPCH (UK); S Pillay, MB ChB, DCH; Y Joolay, MB ChB, FCPaeds, MPhil (Neonatol), Cert Neonatol (SA); N Rhoda, MB ChB, FCPaeds, Cert Neonatol (SA); M S Raban, MB ChB, FCPaeds, Cert Neonatol (SA); A R Horn, MB ChB, DCH, FCPaed (SA), Cert Neonatol (SA); L Tooke MB ChB, FCPaeds, MMed (Paeds), Cert Neonatol (SA)

Neonatal Medicine, Department of Paediatrics, University of Cape Town, South Africa

Corresponding author: M Harrison (m.harrison@uct.ac.za)

Background. Blood transfusions (BTFs) are not without risk and pose a significant financial burden on resource-limited services. In line with current international evidence, the nursery at Groote Schuur Hospital (GSH), Cape Town, South Africa, introduced a restrictive BTF protocol to minimise transfusions and manage costs.

Objective. To determine whether adopting a restrictive BTF policy results in fewer transfusions.

Methods. Data were retrospectively collected on all infants who received BTFs in the GSH nursery over a 6-month period following adoption of a restrictive BTF policy in 2010. BTF figures for a similar time period before the restrictive policy, during 2008, were obtained for comparison.

Results. After introduction of the restrictive BTF policy, 42 of 1097 infants admitted (3.8\%) received a total of 64 BTFs. In comparison, 102 of a total of 940 infants (10.9\%) admitted during a period of the same length before introduction of the restrictive BTF policy received a total of 121 transfusions. Comparison between the number of BTFs administered before and after the restrictive policy showed a highly statistically significant difference ( $p<0.001$ ). The total cost of the blood products used in the two 6-month periods was R91 $870 \mathrm{v}$. R48 640 , based on current prices.

Conclusions. By adopting a restrictive policy, we were able to halve the number of BTFs, reduce risks associated with transfusions, and achieve significant cost benefits. Following evidence-based guidelines results in high standards of care, while also making the most effective use of resources.

S Afr Med J 2013;103(12):916-917. DOI:10.7196/SAMJ.6858

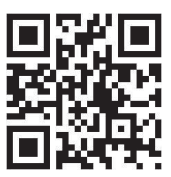

All infants experience a decrease in their haemoglobin concentration after birth, with the risk of anaemia being inversely related to gestational maturity and birth weight. ${ }^{[1]}$ A decrease in haemoglobin is particularly prevalent in infants with a birth weight of less than $1500 \mathrm{~g}$, over half of whom develop anaemia. ${ }^{[2]}$ Three basic mechanisms are responsible for the development of anaemia of prematurity, namely inadequate red blood cell production, shortened neonatal red blood cell lifespan, and iatrogenic blood loss (repeated blood sampling) ${ }^{[3]}$ Babies with anaemia of prematurity often receive multiple blood transfusions (BTFs), with the risks of transmission of infections and iron overload.

Recent evidence has shown that accepting a lower haemoglobin threshold in premature infants before transfusing, i.e. adopting a restrictive BTF policy, resulted in no statistically significant difference in mortality or morbidity compared with infants receiving BTF at a higher, more liberal threshold. ${ }^{[4]}$ Maintaining higher haemoglobin levels results in more infants receiving transfusions, but confers little benefit. ${ }^{[4]}$

The nursery at Groote Schuur Hospital (GSH), Cape Town, South Africa, did not have a formal BTF policy in place before 2010, and transfusion thresholds were often left to the discretion of individual clinicians. After adoption of a restrictive policy (Fig. 1), we conducted a study to determine whether it would reduce the number of BTFs.

\section{Methods}

The GSH nursery is a 75-bed tertiary unit that admits in excess of 2000 infants per year, over 500 of whom have a birth weight of less than $1500 \mathrm{~g}$.
Data were retrospectively collected on all infants who received red blood cell transfusions in the GSH nursery over a 6-month period in 2011 after adoption of a restrictive BTF policy. Data were collected from the blood bank and the patients' medical records and included gestational age, number of BTFs per infant, timing of BTF, clinical status and policy adherence. BTF figures for a similar 6-month period in 2008 before the restrictive policy were obtained for comparison.

Data were analysed with Stata software version 12 (Statacorp, College Station, Tex., USA). Proportions were compared using the chi-square test. Statistical significance was set at $p<0.05$.

Ethical approval for the study was obtained from the University of Cape Town Faculty of Health Sciences Human Research Ethics Committee.

\section{Results}

The restrictive protocol was adhered to in $100 \%$ of cases in which infants received BTFs during the study period. After introduction of the restrictive policy, $42(3.8 \%)$ of a total of 1097 infants admitted received a total of 64 BTFs. Of the transfusions $70 \%$ were to babies with a birth weight of $<1500 \mathrm{~g}$, with most transfusions (39\%) being performed between 14 and 28 days after birth. Apart from one baby who developed necrotising enterocolitis after transfusion, no adverse events were recorded.

In comparison, $102(10.9 \%)$ of a total of 940 infants admitted during a period of the same length before introduction of the restrictive policy received a total of 121 BTFs (Fig. 2).

Comparison between total BTFs administered in 2008 and in 2011 showed a highly statistically significant difference $(p<0.001)$. 


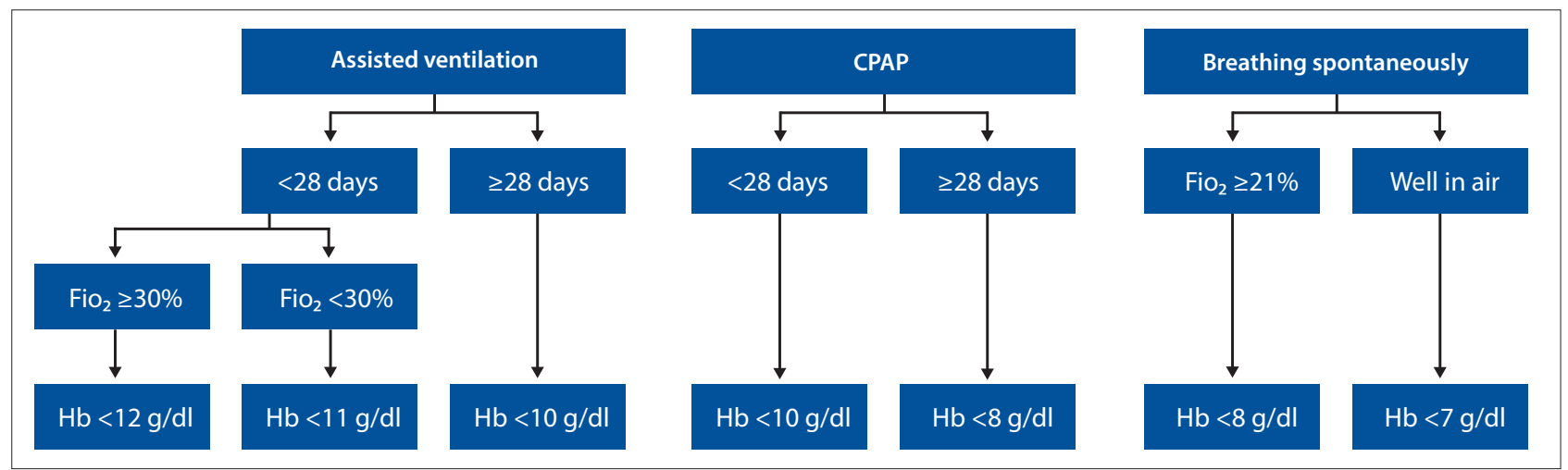

Fig. 1. The Groote Schuur Hospital blood transfusion protocol-guidelines for red blood cell transfusion thresholds for preterm neonates (CPAP $=$ continuous positive airway pressure; $\mathrm{FiO}_{2}=$ fraction of inspired oxygen; $\mathrm{Hb}=$ haemoglobin concentration).

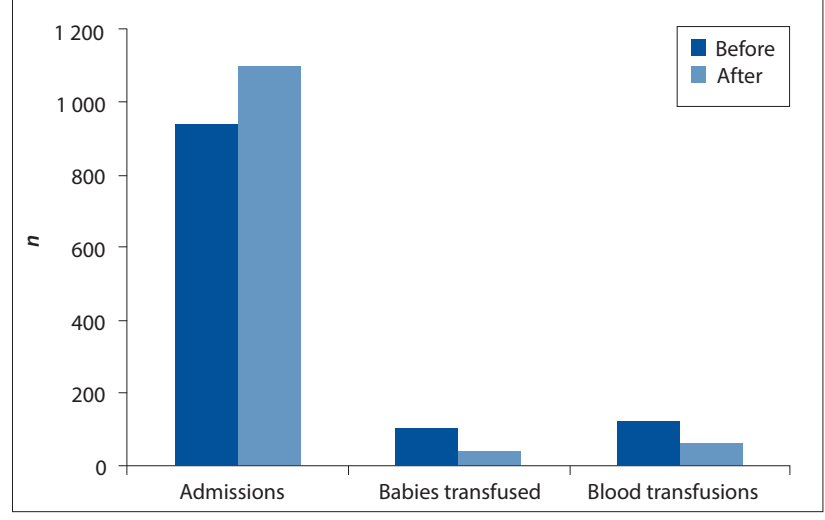

Fig. 2. Comparison of number of blood transfusions and infants tranfsused before and after implementation of the protocol.

Table 1. Cost of blood transfusion products in two 6-month periods

\begin{tabular}{ll}
\hline Total cost & \\
Before protocol implementation & R91 870 \\
After protocol implementation & R48 640 \\
Cost per admission & \\
Before protocol implementation & R97.73 \\
After protocol implementation & R44.34
\end{tabular}

The cost of the blood products used in the 6 months of 2011 was R48 640 compared with R91 870 in 2008, based on current prices (Table 1).

\section{Discussion}

Before introduction of the restrictive BTF policy in the GSH nursery the unit did not have a standardised restrictive protocol to inform BTF decisions, which were left to the discretion of the individual attending doctors. These decisions were often made on an empiric basis, and variations in practice existed between individuals.

In a recent Cochrane review, four trials that enrolled a total of 614 infants compared low (restrictive) with high (liberal) haemoglobin thresholds. There were no statistically significant differences in the combined outcomes of death or serious morbidity at first hospital discharge. ${ }^{[5]}$

This review, coupled with our drive to adopt cost-effective evidence-based guidelines in our unit, resulted in the introduction of a restrictive BTF policy. The adoption of this protocol was consensus driven and had unanimous 'buy in' from all senior clinicians in the unit. The 'buy-in' was evidenced by the fact that the new protocol achieved $100 \%$ compliance during the entire study period.

Evidence-based medicine has been described as 'the conscientious, explicit and judicious use of current best evidence in making decisions about the care of individual patients. ${ }^{[6]}$ Implementation of evidence-based protocols facilitates translation of clinical trials into day-to-day practice, with maintenance of the highest quality and safety of care. Furthermore, resources are allocated cost-effectively.

During the two time periods studied, while the number of admissions remained similar, the number of BTFs administered was almost halved. What was more marked was that the number of infants receiving BTFs was reduced from 102 to 42 , representing an almost $60 \%$ reduction. This resulted in marked cost savings and preservation of scarce blood products, while also decreasing patient exposure to potentially harmful side-effects.

\section{Conclusions}

We have demonstrated that it is possible to achieve $100 \%$ compliance with the commitment of the entire healthcare team, while standardising neonatal care. We were able to halve the number of BTFs and achieve significant cost benefits. Following evidence-based guidelines ensures high standards of care while also making the most effective use of resources.

\section{References}

1. Widness JA. Pathophysiology of anemia during the neonatal period, including anemia of prematurity. NeoReviews 2008;9(11):e520. [http://dx.doi.org/10.1542/neo.9-11-e520]

2. Wardrop CA, Holland BM, Veale KE, Jones JG, Gray OP. Nonphysiological anaemia of prematurity. Arch Dis Child 1978;53(11):855-860. [http://dx.doi.org/10.1136/adc.53.11.855]

3. Strauss RG. Red blood cell transfusion practices in the neonate. Clin Perinatol 1995:22(3):641-655.

4. Kirpalani $\mathrm{H}$, Whyte RK, Andersen C, et al. The Premature Infants in Need of Transfusion (PINT) Kirpalani H, Whyte RK, Andersen C, et al. The Premature Infants in Need of Transfusion (PINT) study: A randomized, controlled trial of a restrictive (low) versus liberal (high) transfusion threshold for extremely low birth weight infants. J Pediatr 2006;149(3):301-307. [http://dx.doi.org/10.1016/j. jpeds.2006.05.011

Whyte R, Kirpalani H. Low versus high haemoglobin concentration threshold for blood transfusion for preventing morbidity and mortality in very low birth weight infants. Cochrane Database Syst Rev 2011, issue 11. Art. No.: CD000512. [http://dx.doi.org/10.1002/14651858.CD000512.pub2]

6. Sackett DL, Rosenberg WM, Gray JA, Haynes RB, Richardson WS. Evidence based medicine: What it is and what it isn't. BMJ 1996;312(7023):71-72. [http://dx.doi.org/10.1136/bmj.312.7023.71]

Accepted 22 March 2013. 